\title{
The Lipogenic Effect of High-Fructose Consumption on NAFLD During Weight Loss
}

\author{
Kelsey B. Stout, Tayler N. Nick, Robert L. Peters, Justin J. Stout, and Joshua S. Wooten
}

Exercise Biochemistry and Physiology Laboratory; Department of Kinesiology and Health Education; Southern Illinois University Edwardsville; Edwardsville, IL

\section{Category: Undergraduate \\ Advisor / Mentor: Wooten, Josh S (jwooten@siue.edu)}

ABSTRACT

Non-alcoholic fatty liver disease (NAFLD) is seen in 30-50\% of type 2 diabetics and $80-90 \%$ of obese individuals. NAFLD is the result of hepatic insulin resistance causing an imbalance between lipogenesis and lipolysis. NAFLD is commonly treated by caloric restriction; however, the influence of continued high-fructose intake on NAFLD during weight loss remains unclear. The purpose of this study was to determine the lipogenic effects of high-fructose consumption on NAFLD during weight loss in obese mice. Male C57BL/ 6 mice were fed either a low-fat (LFD; $10 \% \mathrm{kcal}$ fat) or high-fat (60\% kcal fat) with $20 \%$ fructose solution (HFD+HF) for 8-weeks. Following 8-weeks, HFD+HF were randomly assigned to either LFD or LFD+HF to induce weight loss for 6-weeks. After 6-weeks, plasma was assayed for total cholesterol, triglyceride, glucose and HOMA-IR. Total lipid, triglyceride and cholesterol levels were quantified in hepatic tissues. An ANOVA was used to identify significant $(\mathrm{P}<0.05)$ differences between groups. HFD+HF caused obesity, insulin resistance and hyperlipidemia, as well as NAFLD (Table 1). Following weight loss, the LFD+HF HOMA-IR was similar to LFD mice; however, plasma triglyceride levels remained elevated. Although hepatic mass and percent total lipid were reduced following weight loss, hepatic triglyceride levels in the mice switched to LFD and LFD+HF were 2.2- and 3.0-fold greater, respectively than that found in the LFD control mice. These data suggest that during weight loss high fructose consumption did not impair insulin resistance. Although no difference was observed between either weight loss group for hepatic lipid metabolism and plasma cholesterol; however, hypertriglyceridemia was observed with high-fructose consumption.

Table 1. Whole body and hepatic metabolic characteristics following the 14-week diet intervention.

\begin{tabular}{lcccc}
\hline Variables & LFD $(\mathrm{n}=8)$ & HFD+HF $(\mathrm{n}=8)$ & HFF/LFD $(\mathrm{n}=8)$ & HFF/LFD+HF $(\mathrm{n}=8)$ \\
\hline Body mass $(\mathrm{g})$ & $27.5 \pm 0.6$ & $44.0 \pm 1.5^{*}$ & $31.0 \pm 1.4^{*},+$ & $26.4 \pm 0.8^{+}$ \\
Liver mass $(\mathrm{g})$ & $1.2 \pm 0.1$ & $2.1 \pm 0.2^{*}$ & $1.5 \pm 0.1^{+}$ & $1.3 \pm 0.1^{+}$ \\
Triglyceride $(\mathrm{mg} / \mathrm{dL})$ & $103.8 \pm 5.9$ & $142.3 \pm 15.0^{*}$ & $165.4 \pm 27.0^{+}$ & $81.7 \pm 15.8^{*},+, \neq$ \\
Cholesterol $(\mathrm{mg} / \mathrm{dL})$ & $131.3 \pm 6.4$ & $193.2 \pm 10.9^{*}$ & $118.5 \pm 8.5$ & $168.3 \pm 12.3^{*}, 5^{*}$ \\
Glucose $(\mathrm{mg} / \mathrm{dL})$ & $158.2 \pm 24.3$ & $241.9 \pm 32.2^{*}$ & $127.9 \pm 9.5^{+}$ & $124.6 \pm 6.9^{+}$ \\
Insulin $(\mathrm{ng} / \mathrm{mL})$ & $0.6 \pm 0.1$ & $2.1 \pm 0.9^{*}$ & $0.66 \pm 0.17^{+}$ & $0.69 \pm 0.36^{+}$ \\
HOMA-IR & $5.5 \pm 1.2$ & $32.1 \pm 7.5^{*}$ & $7.3 \pm 2.6^{+}$ & $3.5 \pm 1.9^{+}$ \\
Liver Tg $(\mu \mathrm{g} / \mathrm{mg}$ tissue) & $10.5 \pm 1.6$ & $69.4 \pm 5.7^{*}$ & $23.0 \pm 3.7^{*},+$ & $31.0 \pm 4.6^{*},+$ \\
Liver Chol $(\mu \mathrm{g} / \mathrm{mg}$ tissue) & $10.4 \pm 0.9$ & $8.0 \pm 0.6^{*}$ & $10.9 \pm 0.6^{+}$ & $11.4 \pm 1.0^{+}$ \\
\hline
\end{tabular}

Note. Data are presented as mean \pm SEM. Low-fat diet (LFD); high-fat diet and $20 \%$ fructose solution (HFD+HF); switched from HFD+HF to LFD at 8-weeks (HFF/LFD); switched from HFD+HF to LFD+HF at 8-weeks (HFF/LFD+HF). "Significantly different than LFD; 'Significantly different than HFD+HF; ‡Significantly different than HFF/LFD. 